\title{
GMR
}

$\underline{\text { Short Communication }}$

\section{Fluorescence Image Analyzer - FLIMA: software for quantitative analysis of fluorescence in situ hybridization}

\author{
H.C.M. Silva ${ }^{1,2}$, M.M.C. Martins-Júnior ${ }^{3}$, L.B. Ribeiro ${ }^{4}$ and D.A. Matoso ${ }^{1,5}$ \\ ${ }^{1}$ Laboratório de Evolução Aplicada, Instituto de Ciências Biológicas, \\ Universidade Federal do Amazonas, Manaus, AM, Brasil \\ ${ }^{2}$ Programa de Pós-Graduação em Genética, Conservação e Biologia Evolutiva, \\ Instituto Nacional de Pesquisas da Amazônia, Manaus, AM, Brasil \\ ${ }^{3}$ Empresa Fermento, Manaus, AM, Brasil \\ ${ }^{4}$ Centro de Ciências da Saúde, Coordenação de Medicina, \\ Universidade Federal de Roraima, Boa Vista, RR, Brasil \\ ${ }^{5}$ Laboratório de Citogenômica Animal, Instituto de Ciências Biológicas, \\ Universidade Federal do Amazonas, Manaus, AM, Brasil \\ Corresponding author: H.C.M. Silva \\ E-mail: hallana19@gmail.com \\ Genet. Mol. Res. 16 (1): gmr16019658 \\ Received March 6, 2017 \\ Accepted March 20, 2017 \\ Published March 30, 2017 \\ DOI http://dx.doi.org/10.4238/gmr16019658 \\ Copyright (C) 2017 The Authors. This is an open-access article distributed under the terms of \\ the Creative Commons Attribution ShareAlike (CC BY-SA) 4.0 License.
}

\begin{abstract}
The Fluorescence Image Analyzer (FLIMA) software was developed for the quantitative analysis of images generated by fluorescence in situ hybridization (FISH). Currently, the images of FISH are examined without a coefficient that enables a comparison between them. Through GD Graphics Library, the FLIMA software calculates the amount of pixels on image and recognizes each present color. The coefficient generated by the algorithm shows the percentage of marks (probes) hybridized on the chromosomes.
\end{abstract}

Genetics and Molecular Research 16 (1): gmr16019658 
This software can be used for any type of image generated by a fluorescence microscope and is able to quantify digoxigenin probes exhibiting a red color, biotin probes exhibiting a green color, and double-FISH probes (digoxigenin and biotin used together), where the white color is displayed.

Key words: Cytogenomics; Fluorescence in situ hybridization; Quantitative analysis; Fluorescence coefficient

\section{INTRODUCTION}

The term "cytogenetics" was born by the junction of cytology and genetics, when the theory of chromosomal inheritance based on Mendelian laws and cytological works was proposed. Therefore, when a study is based on an isolated or aggregated chromosome, condensed or distended, with relation to morphology, organization, function, and replication, this study is called cytogenetic study (Guerra, 1988). Cytogenetics can be divided into two branches: classical cytogenetics involving conventional staining techniques, impregnation with silver nitrate, and C-banding and molecular cytogenetics, also called cytogenomics, covering fluorescence in situ hybridization (FISH) and its derivations.

The main technique used in cytogenomics was described in 1986 by Pinkel et al. (1986) and is called FISH. This technique is based on probe marking by fluorochrome through the antigen/antibody bond; these probes are hybridized on the chromosomal DNA marking the region that is demanded and viewed with a fluorescence microscope. Currently, the analysis of FISH images generated by the fluorescence microscope is made only empirically and subjectively, without a quantitative method as a form of analysis. With the development of the FISH technique, the number of analyzed DNA sequences has been increasing, and involves repetitive DNA sequences, single-copy sequences with organizing clusters dispersed or located along the chromosomes.

Because of the lack of a method that allows a quantitative analysis of fluorescent signals generated by the FISH technique, sequences that are dispersed in the genome of organisms (such as transposable elements) can have their location underestimated. This type of analysis is also more difficult to be made in studies where a comparative analysis of the data is necessary. Therefore, the development of a quantitative and objective chromosomal distribution analysis of these sequences is important, and allows the comparison of FISH images. Then, a software that is capable of quantifying the fluorescence generated by the images in fluorescence microscope was created.

\section{MATERIAL AND METHODS}

\section{Library}

GD Graphics Library.

\section{Language}

PHP.

Genetics and Molecular Research 16 (1): gmr16019658 


\section{Information analyzed by the software}

1) Image: generated by a fluorescence microscope when the blades are analyzed. These images must be properly edited and without background (featuring only the chromosomes that will be quantified).

2) Quantity of desired colors for quantification: refers to the amount of probes used for hybridization and thecolors that are shown on the image.

3) Rate tolerance: refers to the accepted level of similarity between colors.

\section{Methods of analysis}

The system breaks the image and analyzes it pixel-by-pixel, recognizing the pixel color and storing the value found. At the end of the analysis all the information is grouped according to the similarity between the colors, and the amount of pixels related to each color is converted proportionally to the coefficient of fluorescence.

\section{RESULTS AND DISCUSSION}

The FLIMA software was developed to quantify FISH images generated by fluorescence microscope in studies where it is necessary a comparative analysis of images, for instance, work that compare numbers of chromosomal markers of a given repetitive element in the DNA of an organism found in different environments. This software may provide a numerical quantitation of which organism presents the higher number of tags. The FLIMA software was created with the purpose of having a coefficient of fluorescence where this coefficient may be used to compare the images. Images that have higher coefficient are those that have more tags. This software can be used for any type of image generated by a fluorescence microscope and is able to quantify digoxigenin probes exhibiting a red color, biotin probes exhibiting a green color, and double-FISH probes (digoxigenin and biotin used together), where the white color is displayed. The FLIMA software quantifies the intensity of the probes, showing the value found in the form of coefficient called coefficient of fluorescence.

For the FLIMA to perform the images analysis (Figure 1), it is necessary that images be edited without background, or must be edited without scales or without any other type of figure that are not part of the chromosomes for analysis (1). Since the image was selected it must be told to software how many colors are expected in the image to be analyzed. Usually for images of FISH we select only 3 colors (but we can select as many colors as the image has): the background of image that is black, the chromosomes stained with DAPI that are blue and markings made on chromosomes that are the colors displayed depending on the probe that is used (2). Then, after the color selection, the tolerance rate must be chosen according to the level of similarity accepted between the colors. The rate of tolerance is used to measure the degree of similarity between the colors in the image; if it is too low the software considers approximate colors as distinct, if it is too high, it considers only the primary colors. As in the image presented by fluorescence microscope shades of red can vary from light or dark pink and red, the recommended rate for analysis is 220 (3). Chosen the first commands to the image, the option "analyze" can be chosen (4). Since the software analyzes the image, the coefficient of fluorescence for each color is exhibited, where the color that displays the probe used is selected (Figure 2).

Genetics and Molecular Research 16 (1): gmr16019658 
Flima-Fluorescence image analyzer

Select the image for analysis.
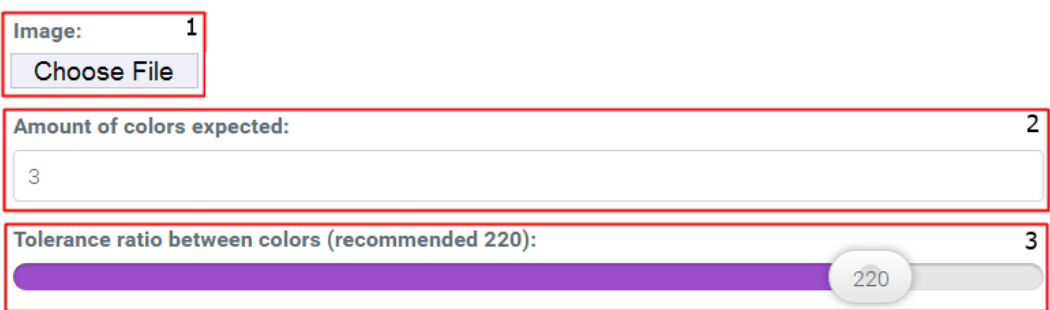

ANALYZE

Figure 1. Initial interface of the FLIMA software.

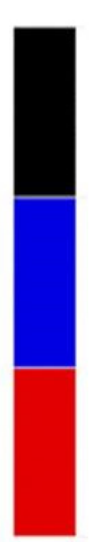

CF

99.952380

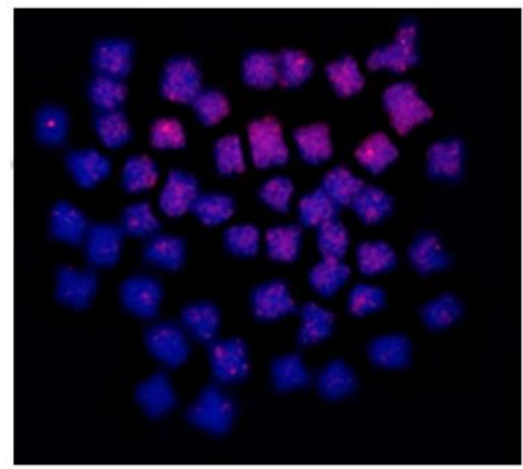

0.0178571

Figure 2. Output interface of analysis of the FLIMA software. The value 99.95 displays the CF (coefficient of fluorescence) to black color. The value of 0.029 is the $\mathrm{CF}$ for the blue color. The value 0.017 is the $\mathrm{CF}$ for the red color. The red color is the hybridized probe on the chromosomes.

The software has been tested in several studies involving FISH data and so far it has been effective in quantifying the marks on the chromosomes. The FLIMA software will be available for commercialization after all processes of intellectual property protection have been made, but their negotiation can be carried out through contact with those responsible for its development.

\section{REFERENCES}

Guerra M (1988). Introdução à citogenética geral. Guanabara 24-35.

Pinkel D, Straume T and Gray JW (1986). Cytogenetic analysis using quantitative, high-sensitivity, fluorescence hybridization. Proc. Natl. Acad. Sci. USA 83: 2934-2938 https://doi.org/10.1073/pnas.83.9.2934. PubMed http:// dx.doi.org/10.1073/pnas.83.9.2934

Genetics and Molecular Research 16 (1): gmr16019658 